\title{
Process Workflow in Crowdsourced Digital Disaster Responses
}

\begin{abstract}
This paper examines the workflow and sense-making activities of digital volunteers, showing how they acquire, assess, process and scrutinise crowdsourced information to warrant confidence that the data satisfies the standard of engagement, production and analysis. We do so by studying a digital disaster response organisation - Humanity Road - through fifteen response operations across thirteen countries using digital ethnography over a period of sixteen months. This paper reports on the findings of this study, using a range of sources such as Skype chat logs, field notes, social media postings, and official documents. This paper also introduces a framework that offers a consistent and structured workflow for the communities of practice related to social media and data aggregation communities within the domain of Digital Humanitarian Networks. Our findings suggest practical implications for both the digital humanitarian organisations and government of the disaster-prone countries.
\end{abstract}

Keywords: Computer Supported Cooperative Work (CSCW), Crisis Informatics, Crowdsourcing, Digital Volunteerism, Disasters, Humanitarian Emergencies.

\section{Introduction}

We are living in a world of emergencies and disasters in which even countries favoured by geography, or advanced technologies are not entirely insulated. Historically, disasters are characterised with the episodic mass influx of goods, services and volunteers that converge to provide relief using collective behaviour and role enactment (Quarantelli and Dynes, 1977; Britton, 1988, 1991). With the advent of mobile technologies along with the development, adoption and increasing accessibility of web 2.0 technologies, the convergence phenomenon that was hitherto observed onsite are now found online by remote digital volunteers (Hughes et al., 2008). These volunteers harness collective intelligence using crowdsourcing to provide situational awareness information for decision making as a form of aid (McEntire, 2004). However, their emergence disrupts the old response playbook of standard operating procedures of emergency response organisations (Tapia, Moore and Johnson, 2013). The rise of such communities, therefore, triggers a renewed academic interest and debates across disciplines.

The focus of this paper is on the established volunteers, unlike most of the existing literature which addresses the activity of spontaneous and unaffiliated volunteers. Specifically, our paper examines the disaster response workflow and crowdsourcing activities of volunteers working under a US-based digital disaster response organisation called Humanity Road (HR). HR is chosen as a case study organisation because of its potentials in adding to our knowledge a peculiar case of how groups are organised, socialised and work together in a virtual space. Furthermore, its work practice also has the potential to highlight how people, organisational culture, and process, as well as 
technological tools and platforms, are entangled with one another. Precisely, this paper seeks to answer the following research question: What activities are being undertaken in processing crowdsourced information?

Thus far, the existing literature concerning the internet-enabled volunteer groups has tended to address the socio-technical challenges associated with Humanitarian emergencies on three broad themes. First, the studies that explore the verification and information processing activities of unaffiliated and spontaneous groups (Starbird, Muzny and Palen, 2012; Starbird, 2013; Tapia and LaLone, 2014; Dailey and Starbird, 2014b; Nhan, Huey and Broll, 2017). Second, works that approach this phenomenon from a more technical perspective related to the development of platforms/tools, and models and framework for extracting disaster information (Imran et al., 2013; Popoola and Krasnoshtan, 2013; Gralla, Goentzel and de Walle, 2015). Finally, studies that seek to understand the work practice of the established groups (Starbird and Palen, 2013). The latter studies focusing on social media and data aggregation communities as an established group have delimited their scope to concentrate mostly on one case study or investigating one disaster type or articulate the usage of a tool/ application over a short period. While insightful, these studies have not comprehensively touched on the activities involved in processing crowdsourced information. This was the motivation behind this study to take a step further and investigate this vital yet underscored aspect of information processing within the social media and data aggregation communities.

As a result, this paper contributes to the existing research by introducing a new framework derived from empirical data developed explicitly for the social media and data aggregation community within the domain of established DVCs. This framework emerged from analysis of data gathered from eight types of disaster covering 13 countries within 16 months. The proposed framework provides a structured, scalable and coherent information workflow on the use of crowdsourcing in humanitarian response operation which forms the theoretical contribution of our paper. By taking a holistic approach to studying different disaster types of various scales, across continents over a more extended period, we differentiate ourselves from prior studies regarding methodology by analysing different data sources. These data sources included field notes from participant observation, digital records of the case study organisation, Skype chat logs and interviews. Employing different data sources allowed us to offer a thick description of how volunteers acquire, process, vet and share actionable information. Consequently, this approach provides a unique contribution to the methodological advancement of disaster research within the Computer Supported Cooperative Work (CSCW) and crisis informatics fields.

The remainder of the paper is organised as follows: Section 2 provides a review of the related studies. Section 3 discusses background and case study context. Section 4 presents data gathering approach, and in section 5 and 6 we present and discuss our findings and its implications. In section 7, we conclude our work. 


\section{Digital Volunteer Communities}

The Literature on digital disaster response is broad-ranging and cuts across disciplines and fields. Our review suggests lack of consensus in naming these Internet-enabled communities from practitioners and academics. For example, terms such as digital volunteer [communities] (Starbird, 2013; Hughes and Tapia, 2015; Kaufhold and Reuter, 2016) digital humanitarians [actor networks] (Meier, 2011; Sabou and Videlov, 2016), volunteer and technical [technological] communities (Gorp, 2014; Weinandy, 2016) have all been used in various studies and across disciplines. This lack of consensus became visible following the publication of Disaster Relief 2.0 where members of digital humanitarian organisations openly disagreed with the authors of the report for giving their various communities a catch-all label as 'volunteer and technical communities' (Standby Task Force, 2011). As such for clarity, we refer to these communities as Digital Volunteer Communities (DVCs).

As mentioned in the introduction, our focus is on the established digital volunteer communities. Gorp (2014), delineates established digital volunteer communities into software platform development communities, mapping communities, expert network communities and social media and data aggregation communities As such, we situated our work within the social media and data aggregation communities by studying the collaborative and social computing aspect of HR volunteers and their crowdsourcing activities.

\subsection{Related Studies}

Previous studies in digital disaster responses have tended to focus on three distinct categories of responders. The first category explores the coordination between digital volunteers and formal/traditional humanitarian relief organisations (Sabou and Klein, 2016). The second segment examines their relationship with emergency management agencies (St. Denis, Anderson and Palen, 2014). The third category deals with the virtual operation support team (VOST) on one hand and digital volunteers on the contrary (St. Denis, Hughes and Palen, 2012; Starbird and Palen, 2013; Palen et al., 2015). Although VOST offers support remotely like any other digital volunteer communities, it is regarded as a distinct entity from the spectrum of the digital volunteers because unlike digital volunteers; its membership came from retired and serving professional emergency management staff who have an internal connection to the response operation (Cobb et al., 2014).

As digital volunteer communities began to mature, scholars have also examined their trajectories and offer insight on how they are changing the landscape of disaster response. For example, Palen et al. (2015) explore the path of Humanitarian OpenStreetMap through the lens of two major disasters and provide a glimpse of how it organises its activities. Similarly, Starbird (2013) has explored the information processing activities of $\mathrm{HR}$ volunteers by examining the management of information using a specific case study of Sacré Coeur hospital following Haiti 2010 earthquake. On the other hand, Starbird \& Palen (2013) provide an insight into the organisational develop- 
ment and work practice of HR where they traced its origin, identity negotiation, membership and the nature of its work. Nonetheless, these studies have limited scope with regards to the contexts, the use of tools and the duration upon which the response lasted. For instance, in the case of the Peru Earthquake, the entire response operation lasted for 3 hours and involved eight volunteers. As such, our paper, builds on such contributions by observing 15 response operations across 13 countries over a period of 16 months, to provide a holistic understanding of how volunteers acquire, assess, process and scrutinise crowdsourced information. The exploration of such activities will, therefore, contribute to the better understanding of the behind-the-scenes information processing activities of DVCs. Such findings will thus serve as a benchmark for evaluating whether the kind of information services these communities are providing satisfied standards of engagement, production and analysis.

\section{Background and Case Study Context}

HR uses Skype as its core platform for coordinating its activities. Precisely, these activities are organised mainly in 4 different Skype 'windows' named as HR Café, HR Urgent Events, HR Useful Links, and HR Work Diary. HR Café is a window in which volunteers are using as a resort for socialising and exchange pleasantries. HR Urgent Events window is the central hub where volunteers coordinate and monitor the ongoing event around the world. HR Useful Links window is another resort where volunteers post exciting life hacks, tips, notes, and links while HR Work Diary is serving as a platform for volunteers to stop in and drop a note on what they have been working on so that other team members can stay informed. In addition to these 4 main windows HR have separate windows for committee meetings, special projects, internal drills and training. Again, HR creates event specific window whenever it is responding to the significant catastrophe.

\section{$4 \quad$ Methodology}

Our approach to understanding this collaborative and social computing phenomenon is through virtual ethnography. Recent studies in CSCW and Crisis informatics has promoted the use of such methods (Ducheneaut, Moore and Nickell, 2007; Irani, Hayes and Dourish, 2008; Starbird and Palen, 2013).

We used field notes from participant's observation, digital records of the case study organisation, Skype chat logs and interviews as our primary source of data for this research. The first author participated in thirty meetings in which twelve related to strategic and operational aspects of the organisation, while the remaining eighteen were associated with volunteer training. Drawing from the theory of role and self (Gold, 1958), the first author takes the role of participant-as-observer where he spent the time to take part in the real disaster response.

HR also allowed us to use Skype chat log with the condition that all personally identifiable information will be anonymised unless a volunteer expressly agreed to be made 
public. As such, our review of the Urgent Event Window chatter involves only the response operations that our first author participated or observed. Specifically, the review coverage spans from 15th February 2016 to 22nd May 2017.

We also used Skype to conduct semi-structured interviews with 7 volunteers to find out detailed information about the work process, standards, collaboration, trust, use of ICT tools and protocols associated with the response operation which lasted between 60 to 120 minutes.

This paper uses 15 selected digital disaster response operations undertaken by HR as its primary unit of analysis. To choose those responses, we consider Fritz (1961, cited in Kreps, 1984) core properties of disasters - events, social units, response - as our basis for case identification and selection. Accordingly, we attempted to cover a broad range of disasters (events) - dam spillage, earthquakes, explosions, flooding/landslide, hospital project, severe weather, tornado, wildfires - to encompass a comprehensive set of workflow activities from different emergency response operations. Also, our case selection covers 13 countries (social units) drawn across 6 continents - Africa, Asia, Europe, North America, South America, Oceania - with a different type of activation (response) as shown in Appendix 1. Our basis for selecting these events is to have insight into whether there is a difference of approach or procedures across disaster types or countries in digital disaster response work.

Our approach to data analysis is qualitative. As such, we draw from Corbin and Strauss' (1990) procedures, and previous examples in CSCW papers (Procter et al., 2016; Farshchian, Vilarinho and Mikalsen, 2017). Following that, we review and analyse field notes, chats log, interview transcripts, and digital records iteratively and inductively to find recurrent themes. At the first instance, we began our analysis by making sense of the entire Skype chatter covering the 15 major response operations, thereby extracting themes and subthemes based on our research objectives. Later, we compared notes from our field notes, and other sources mentioned earlier to understand the organisational approach to response coordination.

\section{$5 \quad$ Findings}

The purpose of this study was to find out the type of activities involved in processing crowdsourced information. As such, we begin by discussing the process workflow that emerged based on repeated occurrence across a range of disasters during our sixteen months' observation.

Our observation along with a careful review of Skype chatter across a range of disasters revealed an implicitly structured workflow. This workflow starts with Monitoring and continues with Activation, Listing, Listening \& Verification, Amplification and ends with Reporting. We categorised these activities into six segments in which each phase is distinct, with a related set of tightly constrained interdependent activities that are repeatedly present in every response operation.

In what follows is the explanation of our findings on the nature of cooperative work of the HR digital response partly drawn from the Skype chatter during a response operation. The portion of the Skype chatter we illustrate here usually starts with a date 
and time stamp ([12/03/2016, 20:17:29]) followed by the name of the volunteer that mostly begins with a prefix HR. The Skype chatter portion also include emoticons that volunteers usually inserts in between the message they want to pass across.

\subsection{Monitoring}

The foundation upon which digital disaster response is built lies in receiving credible information about the sudden onset or arrival of slow-moving disaster. Obtaining such information entails the beginning of digital disaster response. The HR initiation stage for the response, therefore, begins at the point of watching for disasters to unfold: we refer to this as the Monitoring phase. Our finding reveals HR volunteers employ a different range of applications and platforms for receiving an instant push notification. As such websites like the Global Disaster Alert and Coordination System (GDAC), U.S. Geological Survey (USGS), National Hurricane Centre (NHC) and Pacific Disaster Centre (PDC) remained the most preferred source of news for HR volunteers. Furthermore, breaking news websites and media outlets are considered as secondary sources. This is because HR volunteers have learnt over the years that "some of these media sources have the history of publishing information very fast but often get their facts wrong" (Interview, President HR). HR volunteers, therefore, prefer to subscribe to electronic notification systems (ENS) of GDAC, USGS, NHC and PDC among others. By their subscription, these volunteers receive an SMS or email every time a disaster happens or is about to happen. Notification received via these websites sometimes offer a snippet of the disaster impact that helps volunteers to start preparing for a response. The following piece from the Skype logs illustrates how this volunteer received an instant alert and was announcing to the Urgent Event window about the sudden onset of Earthquake in Alaska:

[12/03/2016, 20:17:29] HR Nicholas Lawson: I just got a phone notification of a magnitude 6.4 EQ near Atka, Alaska - alert doesn't give depths - will look for that - EQ occurred at 18:06 UTC **

Figure 1. Snapshot of Skype logs extracts 1. - Illustration of notification alert. As soon as information of such nature is posted into the Urgent Event Window, available volunteers that are hanging around in the Café will report to the window where a series of discussion will begin. The outcome of such meeting will determine whether to keep monitoring the situation or activate the Disaster Desk in which active and inactive volunteers will be invited. Activating Disaster Desk for disaster response signifies the end of the first phase and the beginning of the second phase.

\subsection{Activation}

In disaster response parlance, activation levels define the kind of response emergency workers will offer during any catastrophe. For instance, Stage 1 (Green) activation is designated for local events that are smaller in proportion in which information is limited or events whose level of impacts is yet to be ascertained. In that instance, volunteers will data mine social media for urgent needs and route it to those offering help and vice versa. Stage 2 (Yellow) activation is when the event is severe and humanitarian emergency organisations are overwhelmed and could not be able to respond to urgent needs 
promptly. In these circumstances, HR volunteers undertake 'general monitoring' and collaborative authoring of a 'situation report' (SitRep). During stage 2 (Yellow) activation, all regularly scheduled meetings and training sessions of HR may be temporarily disrupted. Stage 3 (Code Red) is designated for massive catastrophe with mass fatalities usually needing international aid.

For HR, Code Red activation needs a collective effort from both active and inactive volunteers. Active volunteers are HR members who are active in all the four main HR Skype windows while inactive members have access to all the windows but resurface to give help when HR social media incident commander sends an activation invitation.

Immediately after activating to Stage 3, HR Incident Commander will send text messages and formal (email) invitation to both active and inactive volunteers requesting for their help. The email will typically have a brief overview of the catastrophe, the nature of response HR will offer, and a call to report to Urgent Event Window or a choice for amplifying HR work through social media platforms (if the volunteer may not have time to take part in the response operation). Moreover, the Incident Commander will also post the same announcement in the Urgent Event window and will create an eventspecific window for managing the response. The following excerpt illustrates how an Incident Commander posted a statement to the Urgent Event Window:

[03/10/2016, 17:50:01] HR Javon Malone: (star) Attention team, we anticipate activation this afternoon for Hurricane Matthew. This is a very large storm that will likely impact multiple countries. The DDWG is preparing for our response now. This will likely be a Yellow and quite possibly Red event. We ask that if you are able to sta it clearing some time in your schedule the next 3-4 days to support our activation. We have a window set up and gave designated this event as Operation Atlantis. Thank you. (f) (f) (f)

Figure 2. Snapshot of Skype logs extracts 2. - Activation announcement.

In the above extract, the Incident Commander started his post with the star (star) emoticons signifying the start of the new event and ended it with three flower emoticons [(f) (f) (f)] which denote an end of the message. We can deduct from the statement that HR has been watching the forecast. The Incident Commander also mentioned DDWG (the Disaster Desk Working Group) readiness to support the event. The DDGW is a subteam of active volunteers that have separate windows in which they meet and decide on matters related to disaster response.

From the preceding, we can see that activation phase began as soon as monitoring phase ends and the phase entails the decision-making process and the nature of the response HR will be providing.

\subsection{Listing}

Following the activation of disaster by the social media incident commander, volunteers will typically start announcing their availability. At this stage, available volunteers will be asked to perform Listing activities. Listing in this context refers to crowdsourcing the most valuable information on the internet that will guide the response and coordination of the disaster. Such activities include researching keywords and event hashtags 
that will be used in data mining in Scanigo - a social media analytic toolkit for extracting disaster information. This toolkit organises tweets into categories, helps in reducing Twitter noise, and identifies likely relevant tweets.

Some volunteers will also be asked to find a country's emergency numbers for dispatch, fire, ambulance, and police while others will be working on listing websites that are responsible for providing official updates. For example, HR co-ordinators will be interested in finding sites, social media handles, location, phone numbers, and emails of country's emergency management agencies. Volunteers will also be asked to offer information related to regional and local organisations of the disaster-affected region.

Listing activities also entail finding social media handles of traditional relief organisations like Save the Children, Doctors without Borders, and Red Cross. HR also encourages its volunteers to provide social media handles and contacts of critical infrastructure companies associated with communication, road, and airport among others. Additionally, volunteers will be expected to provide information on websites, contact address, location, social media handles of organisations dealing with disability, accessibility and functional needs (DAFN) and a host of other interest groups depending on the nature of the catastrophe and the country.

Unlike the 'Monitoring' and 'Activation' phases in which the activities are in sequence, the Listing phase often connects to the next phase (Listening and Verification) in a back and forth manner depending on the nature of the catastrophe. If the situation is not a complex and does not need major response effort, the workflow is a straightforward activity. As such, the sequence will be from 'Listing' to 'Listening and Verification' phase. However, if the response initially pertains only to one area but later the situation keeps magnifying, and various regions are added to the emergency declaration list, then the activity will continuously be in a cyclical iteration between the two phases as illustrated in Appendix 2. Moreover, our observation reveals another phase called 'Reporting' (see section 5.6) also connects directly to 'Listing' phase during major events. The two dotted arrows in Appendix 2 - Figure 3 becomes active whenever the event is declared to be a major one as such there is a need for updating the 'Listing' resources whenever the emergency declaration covers new areas as the event keeps unfolding.

In summary, 'Listing' phase involves the use of manual and automated tools to data mine actionable information that helps people survive, sustain, and reunite. Specifically, during this phase, the volunteers will be looking for information such as emergency numbers for ambulance, fire service, and police that will help people and animals survive, sustain and reunite. Volunteers will also search for contacts including website links, social media handles, telephone numbers, location for organisations, aid agencies, and local support groups among others.

\subsection{Listening and Verification}

Unlike the previous activities that occur in sequence, our observation across disasters reveal 'Listing' and 'Listening \& Verification' are iterative, interrelated and distinct activities that sometimes happen simultaneously. In the context of HR, listening entails data mining social media for finding damage reports and urgent needs such as a request 
for help, evacuation, medical supplies, missing person or information about reunification centres among other things.

Also, listening includes monitoring the Scanigo platform, searching for isolated and disadvantaged communities as well as tracking the activities of other digital volunteers and aid agencies. It also involves listening to online Emergency Telecommunications groups such as Ham Radio and First Response Radio Team (FRRT).

As soon as a volunteer uncovers information arising from the listening activities, the next action is to post the information to the Urgent Event window for verification. Volunteers will then begin to triangulate the information, in some instances to revert to the original source or contact partners for further clarification or affirmation. If the exercise did not yield any positive outcome, the issue would be put on hold, and other volunteers will continue to monitor the situation. However, if something positive came out of it, a decision may be taken to amplify the information and record the activity on the SitRep. This act of collective effort and decision making is what we refer to as Verification.

Previously, we have shown 'Listing' and 'Listening \& verification' workflow move either in sequence or in back and forth manner; however, in this phase, the workflow sequence moves in the forward direction to the next phase called 'Amplification'. In addition to that, during major events, our observation reveals a linkage between the 'Reporting' phase and 'Listening \& Verification' phase. This activity evolves when the situation warrants writing a series of SitReps. As such, volunteers' co-authoring reports will be transferring vetted information from the Skype response window and keep adding them to the Google Docs for authoring the SitRep. An illustration of workflow is in Appendix 2 - Figure 4.

The figure shows the process workflow at 'Listening \& Verification phase' with the 'Listing' as a preceding activity and 'Amplification' as the next stage in the process. The figure also shows a dotted arrow from 'Reporting' phase linking to the 'Listening $\&$ Verification' phase. Listening and Verification are ongoing activities until when the disaster desk Incident Commander announce volunteers to stand down.

\subsection{Amplification}

Amplification comes into effect as soon as volunteers verify or track critical information which needs to be routed to emergency management organisations or disasteraffected communities. The act of sharing such information is what is called amplification. More precisely, amplification involves sharing verified official information sourced from emergency management organisation in charge of response operation and other traditional humanitarian relief agencies to the public. It also involves routeing urgent needs of those in needs of emergency management agencies and humanitarian aid organisations. Amplification also includes posting survival tips and reassurance messages by HR volunteers through official and individual volunteers' social media accounts. It also involves urging the public to be cautious of sharing photos and location of the emergency responders while working at public safety event.

From the context of response workflow, the sequence that leads to 'Amplification' phase starts from 'Listening \& Verification', and the workflow continues to the Reporting phase from Amplification phase. 
In summary, amplification comes into effect whenever volunteers vetted and approved the information to be shared with the public. This vetting and approval are undertaken at the 'Listening \& Verification' phase. As such, the nature of the workflow starts with 'Listening \& Verification' phase and is a one-way continuous activity until the disaster response is over. The rate at which HR volunteers amplify information depends on nature, impact, and the affected area(s). It is worthy to note HR amplify information across its social media platforms and to encourage volunteers to also amplify the same information through their social media handles.

\subsection{Reporting}

The Reporting phase comes into effect depending on the nature of the disaster. If the catastrophe needs long hours of response, then HR volunteers will start co-authoring SitRep at the same time while amplifying and this will be going on until the response is over. Sometimes as the situation keeps evolving, volunteers will keep updating the resource list. For example, it is possible in the first instance, a state of emergency declared earlier covers only one County, but as the situation unfolds, the declaration will also include nearby counties or regions. As such, some volunteers will update the listing resource while others are working on the main SitRep. Also, as the situation keeps evolving, Listening and Verification will also be going on simultaneously with the Reporting as illustrated in Appendix 2 - Figure 5. On the other hand, if the event does not require SitRep, reporting will only be in the Urgent Event Window which will later culminate into standing down from the response operation. The figure depicts the workflow where volunteers working on the Reporting phase can go back either to Listing or Listening \& Verification Phase. For example, the situation might warrant the need for the original lists to be updated when the disaster covers more regions.

Our observations also reveal the use of four primary tools/platforms in mediating the cooperative work of producing a report popularly called SitRep. First, the use of Skype as a central platform where all the chatter for mediation, verification, and sense-making takes place. Second, the use of Google Sheet where all the event status such as instructions, guides and tip sheet, are filed and archived. Third, the use of Google Docs in which volunteers cooperatively work to develop the SitRep which mainly assembles information and updates that will help people and animals in disaster to survive, sustain and reunite. Fourth, the use of Scanigo social media analytic tools for filtering, categorising and ranking torrents of tweets to reduce the time taken for data mining Twitter noise. Additionally, volunteers using the Firefox browser can appropriate HR's plugin while responding to the catastrophe. The custom-made plugin provides a handy dashboard where volunteers could easily find pre-written searches, guides, tips, a list of embassies, twitter lists, emergency numbers and loads of useful information.

From our findings, partners such as United Nation Office for the Coordination of Humanitarian Affairs (UNOCHA), FEMA, Americares, and Cisco TacOps uses HR SitRep for situational awareness and decision making associated with communication, funding and cooperation. SitRep also guides partners to know who is doing what and where among other aid workers and relief agencies. 
In brief, HR digital disaster response workflow starts with a sequence of activities evolving into six distinct phases. The first three phases - monitoring, activation and listing -, developed in series while there is a continuous looping of actions between listing and listening \& verification phase and the sequence will continue from listening $\&$ verification phase through amplification to the reporting phase. Next is a back and forth iteration at reporting stage that came into effect from amplification. At the reporting phase, a series of this back and forth iteration centres around listing and listening \& verification phases depending on the nature of the catastrophe.

\section{Discussion and Implications}

This study investigated HR's activities in acquiring and verifying crowdsourced information. Building upon the analysis section explained above; this paper goes on to discuss the findings' relationship with the research question.

\subsection{Digital Disaster Response Process Workflow}

Regarding our research question that sought to answer the type of the activities involved in processing crowdsourced information, our findings uncover 6 distinct, repeatable patterns associated with every disaster response cycle we studied. This pattern evolves in phases as follows: 1) Monitoring, 2) Activation, 3) Listing, 4) Listening \& Verification, 5) Amplification, and 6) Reporting. As seen in Appendix 2, the response pattern shows a combination of linear as well as reciprocal workflow based on the magnitude of the disaster.

Accordingly, based on the results of the study in the investigated contexts, this paper proposes an analytical framework that offers a fresh perspective and deeper insight into the activities of the digital humanitarian response process workflow (Appendix 2 - Figure 6)

As it is seen in Appendix 2 - Figure 6, the proposed analytical framework holds the essential steps to understanding digital disaster response. These steps were carefully analysed and cross-checked across 8 disaster types in 13 countries during 15 response operations as explained in the analysis section. The conceptualisation of these phases gives an exciting insight into the activities of HR. As such, while our model is highlevel enough to allow academics, practitioners and system designers to make sense of the process workflow in HR, at the same time, it provides detailed interpretations of each step in the process and how they are dynamically and reciprocally related to one another. This makes our model flexible in its scalability that can be followed as a roadmap and applied in different contexts across diverse disaster types with different with differing impacts and scales.

This scalability covers the disaster type, impact, affected region and the country. By disaster impact we refer to the number of causalities, property and infrastructural damages. The affected region could be isolated communities or in urban areas. Countries could take the form of developed or developing nations since HR response lies entirely on the information available online and the emergency response system of the country in question. Keeping this explanation in mind, the workflow moves in sequence from 
monitoring to reporting phase when the activation is in Green or Purple - an activation designated for special projects. However, once the activation is Yellow or Red, Listing, Listening \& Verification, as well as Reporting (as shown in Appendix 2 - Figure 6), will be in the continuous iteration until the operation ends. The dotted arrows along with numbers $(1,2,3)$ illustrate the back and forth processual iteration of the workflow.

By proposing the analytical framework, we generalise from empirical statements (as inputs to generalising) to theoretical statements (as outputs of generalising) (Lee and Baskerville, 2003). Furthermore, structuring, evaluating and verifying this model upon various heterogeneous cases and contexts across both developed and developing countries generalise our model to provide insight into the domain of social media and data aggregation communities within the digital volunteer communities. Following on from this, we now turn to examine the collaborative and social computing activities performed by volunteers during each response operation. These areas include: tools manipulation, task organisation, communication style, and the group shared awareness:

Tool manipulation: The response workflow mentioned earlier also reveals how HR volunteers are manipulating tools to produce contents. While the use of such devices is common knowledge, but how HR team is shaping the tools in the context of disaster response is what makes this interesting. For example, during a typical response, the HR team uses Skype for coordination, Google Docs for collaborative authoring, Google Sheets as information and project management/reference manual, and Scanigo for listening to social media postings. In other instances where the response dictates the need for more training to volunteers or briefing (like the Burundi Hospital response), HR will use a Google slide deck to visually present ideas for volunteers. As such, the ability to combine automation alongside manual work such as the use of Scanigo or switching from one platform as well as searching or posting information using a mobile phone or desktop computer to another platform suggests a combination of creativity, experience and skills.

Task Organisation: Observing HR workflow also enabled us to understand how they organise activities by dividing the task among themselves. For example, in the Listing phase, the work involves finding health facilities, embassies, telecommunication companies, emergency management organisations and relief agencies among others. At Listening \& Verification, some volunteers' role will be listening to isolated communities, others on reunification information and another team will be charged with data mining urgent needs. When it comes to Amplification, some volunteers will take the role of posting information to HR tweeter account, while others will work on Facebook or Instagram. But how they work out what to do and who to do what while responding depends on the available volunteers, their skills, knowledge of contexts, and the nature of the response - slow moving or sudden.

Communication Approach: Another insight associated with our findings involves the nature of information exchange where the communication is characterised using abbreviations, emoticons, typing errors, hedging, emphasis and terminologies. Use of acronyms such as BRB for 'be right back', EQ for an earthquake, SitRep for situation reports and TC for tropical cyclone among others is replete in all their communication. Likewise, HR standardises the use of some selected emoticons for shared awareness among volunteers. In some circumstances, information exchange in the Urgent Event 
window is characterised by spelling mistakes which can be likened to the altruistic urge and pressure to help. Our observation also reveals the manifestation of caution such as 'heads off' or 'unverified' while posting information to prepare the mind of fellow volunteers.

Group Shared Awareness: The concept of shared awareness within a group is well established in CSCW literature (Borghoff and Schlichter, 2000). As such, our observation noted with keen interest how 'Reporting' in the form of 'morning summary', 'evening summary' and 'end of the day summary' among others is playing a significant role in a response operation. During response operation, the HR team will assign a member to take the role of giving a summary and posting it to the Urgent Event window. The essence is to make everyone aware of the ongoing response since volunteers' work based on their conveniences.

\subsection{Practical Implication}

The main contribution of this paper is that it has generated essential and valuable insights into the phenomenon of digital volunteerism about the social media and data aggregation communities. As a result, the findings of our study suggest implications for practice for digital humanitarian organisations, emergency management agencies, Governments of disaster-prone countries as well as directions for future research. This paper has sketched out how the crisis information data is monitored, processed, managed, verified and reported. Based on the empirical findings we proposed a new framework to show the order of activities being undertaken in preparing crowdsourced information. While this framework is the first of its kind to represent the big picture, it provides practitioners, policymakers and system developers with a roadmap currently being followed so they can more seriously consider their contributions to this workflow to improve this process further and more efficient.

Moreover, our findings have important implications for the Governments of disasterprone countries. For example, our inclusion of the different type of disasters and emergencies across countries has revealed the dearth of information that HR makes use of to monitor the official response from the Governments of the affected countries, especially from the developing nations. Our experience in Sri Lanka, Burundi, Peru and Fiji have revealed the scarcity of information about websites, social media handles, and emergency numbers of the emergency management organisations where volunteers can find verified official information to route that information to the people. As such, if countries will make information available online by creating websites and having official social media handles, it will enable volunteers to follow and relay official details to those in needs. Likewise, digital disaster response organisations such as HR could also develop a pre-made compendium of information resource list of disaster-prone countries before the sudden onset of disasters. Information resource list is a directory that holds information such as emergency numbers, websites, locations, maps, social media handles of fire, ambulance, police, airports, transportations and emergency response organisations. Developing such compendium will enable organisations such as HR to cut time in crowdsourcing the list at the 'listing' stage while responding to disasters as illustrated in section 5.3. By so doing, the HR process workflow could be 
reduced to monitoring, activation, listening and verification, amplification as well as reporting.

\section{Conclusion}

This study contributes to the Computer Supported Cooperative Work (CSCW), Crisis Informatics, Information Systems for Crisis Response and Management (ISCRAM), and Disaster field in two ways: theoretically and methodologically.

As to the theoretical contribution, this study proposes a new analytical framework outlining the various stages/activities involved in the digital disaster response information workflow for the social media and data aggregation communities. Comprehensively derived from the empirical data, this framework contains the critical components that could potentially signal the emergence of new models for DVCs with regards to the social media and data aggregation Community. Our model evolved from diverse types of disasters with different scales and is flexible enough to support multiple scenarios of similar kinds and to be adapted to various disaster types and used by Social Media and Data Aggregation Communities with comparable characteristics in different countries and settings.

Regarding the methodological contribution, this study is among the few that attempts to empirically and comprehensively understand and shed light on the information processing workflow. As mentioned previously, this study employed various data collection methods from 8 disaster types in 13 countries, across 6 continents, covering both developing and developed nations using virtual ethnography over the period of 16 months. To the best of our knowledge, this methodological approach is the first of its kind in this area with regards to the DVCs focusing on social media and data aggregation communities. Taking this approach has provided a holistic understanding of the behind the scene processes and measures in digital disaster response. 


\section{References}

Borghoff, U. M. and Schlichter, J. H. (2000) Computer-Supported Cooperative Work, Computer-Supported Cooperative Work. doi: 10.1007/978-3-662-04232-8_2.

Britton, N. R. (1988) ‘Organised Behaviour in Disaster: A Review', International Journal of Mass Emergencies and Disasters, 6(3), pp. 363-395.

Britton, N. R. (1991) 'Permanent Disaster Volunteers: Where Do They Fit?', Nonprofit and Voluntary Sector Quarterly, 20(4), pp. 395-414. doi: 10.1177/089976409102000404.

Cobb, C., McCarthy, T., Perkins, A., Bharadwaj, A., Comis, J., Do, B. and Starbird, K. (2014) 'Designing for the Deluge: Understanding \& Supporting the Distributed, Collaborative Work of Crisis Volunteers', Proceedings of the Conference on Computer Supported Cooperative Work (CSCW), pp. 888-899. doi: 10.1145/2531602.2531712.

Corbin, J. and Strauss, A. (1990) 'Grounded theory research: Procedures, canons, and evaluative criteria', Qualitative sociology. Available at: http://link.springer.com/article/10.1007/BF00988593 (Accessed: 1 June 2017).

Dailey, D. and Starbird, K. (2014b) 'Visible skepticism: Community vetting after Hurricane Irene', in ISCRAM 2014 Proceedings - 11th International Conference on Information Systems for Crisis Response and Management, pp. 777-781.

St. Denis, L. A., Anderson, K. M. and Palen, L. (2014) 'Mastering Social Media : An Analysis of Jefferson County' s Communications during the 2013 Colorado Floods', Iscram, (May), pp. 737-746.

St. Denis, L. A., Hughes, A. L. and Palen, L. (2012) 'Trial by Fire: The Deployment of Trusted Digital Volunteers in the 2011 Shadow Lake Fire', 9th International ISCRAM Conference, (April), pp. 1-10.

Ducheneaut, N., Moore, R. J. and Nickell, E. (2007) 'Virtual Third Places': A Case Study of Sociability in Massively Multiplayer Games*', Computer Supported Cooperative Work, 16, pp. 129-166. doi: 10.1007/s10606-007-9041-8.

Farshchian, B. A., Vilarinho, T. and Mikalsen, M. (2017) 'From Episodes to Continuity of Care: a Study of a Call Center for Supporting Independent Living', Computer Supported CooperativeWork (CSCW), (26), pp. 309-343. doi: 10.1007/s10606-017-9262-4.

Fritz, C. E. (1961) Disasters. In Social Problems. Edited by R. Merton and R. Nisbet. New York: Harcourt, Brace \& World pp. 651- 694.

Gold, R. L. (1958) 'Roles in sociological field observations', Social forces. JSTOR, 36(3), pp. 217-223. doi: 10.2307/2573808.

Gold, R.L., 1958. Roles in sociological field observations. Social forces, pp.217-223.

Gorp, A. F. Van (2014) 'Integration of Volunteer and Technical Communities into the Humanitarian Aid Sector: Barriers to Collaboration', Proceedings of the Information Systems for Crisis Response and Management (ISCRAM), (May), pp. 620-629.

Gralla, E., Goentzel, J. and de Walle, B. V (2015) 'Understanding the information needs of field-based decision-makers in humanitarian response to sudden onset disasters', Proceedings of the 12th International Conference on Information Systems for Crisis Response and Management (ISCRAM), pp. 1-7.

Hughes, A. L., Palen, L., Sutton, J., Liu, S. B. and Vieweg, S. (2008) 'Sight-seeing in 
disaster: an examination of on-line social convergence', in Proceedings of the Information Systems for Crisis Response and Management Conference (ISCRAM 2008), pp. 44-54.

Hughes, A. L. and Tapia, A. H. (2015) 'Social Media in Crisis: When Professional Responders Meet Digital Volunteers', Journal of Homeland Security and Emergency Management, 12(3), pp. 679-706. doi: 10.1515/jhsem-2014-0080.

Imran, M., Castillo, C., Diaz, F. and Meier, P. (2013) 'Practical Extraction of DisasterRelevant Information from Social Media', in Proceeding of International World Wide Web Conference Committee (IW3C2) WWW 2013 Companion, May 13-17, 2013, Rio de Janeiro, Brazil. ACM 978-1-4503-2038-2/13/05, pp. 1-4.

Irani, L. C., Hayes, G. R. and Dourish, P. (2008) 'Situated practices of looking', in Proceedings of the ACM 2008 conference on Computer supported cooperative work - CSCW '08. New York, New York, USA: ACM Press, p. 187. doi: 10.1145/1460563.1460592.

Kaufhold, M. A. and Reuter, C. (2016) 'The Self-Organization of Digital Volunteers across Social Media: The Case of the 2013 European Floods in Germany', Journal of Homeland Security and Emergency Management, 13(1), pp. 137-166. doi: 10.1515/jhsem-2015-0063.

Kreps, G. A. (1984) 'Sociological Inquiry and Disaster Research', Annual Review of Sociology, 10(1), pp. 309-330.

Lee, A. A. S. and Baskerville, R. R. L. (2003) 'Gerneralizing Generalizability in Information Systems Research', Information Systems Research, 14(3), pp. 221-243. doi: 10.1287/isre.14.3.221.16560.

McEntire, D. A. (2004) The status of emergency management theory: Issues, barriers, and recommendations for improved scholarship. University of North Texas. Department of Public Administration. Emergency Administration and Planning.

Meier, P. (2011) 'New information technologies and their impact on the humanitarian sector', International Review of the Red Cross, 93(884), pp. 1239-1263. doi: $10.1017 / \mathrm{S} 1816383112000318$.

Nhan, J., Huey, L. and Broll, R. (2017) 'Digilantism: An analysis of crowdsourcing and the Boston marathon bombings', The British Journal of Criminology. Oxford University Press, 57(2), pp. 341-361. doi: 10.1093/bjc/azv118.

Palen, L., Soden, R., Anderson, T. J. and Barrenechea, M. (2015) 'Success \& Scale in a Data-Producing Organization: The Socio-Technical Evolution of OpenStreetMap in Response to Humanitarian Events', Proceedings of the ACM CHI'15 Conference on Human Factors in Computing Systems, 1, pp. 4113-4122. doi: 10.1145/2702123.2702294.

Popoola, A. and Krasnoshtan, D. (2013) 'Information verification during natural disasters', Proceedings of the 22nd international conference on World Wide Web, pp. 1029-1032. doi: $10.1145 / 2487788.2488111$.

Procter, R., Wherton, J., Greenhalgh, T., Sugarhood, P., Rouncefield, M. and Hinder, S. (2016) 'Telecare Call Centre Work and Ageing in Place', Comput Supported Coop Work, 25(79), pp. 9242-5. doi: 10.1007/s10606-015-9242-5.

Quarantelli, E. L. and Dynes, R. R. (1977) 'Response to Social Crisis and Disaster', Annual Review of Sociology, 3(1), pp. 23-49. doi: 10.1146/annurev.so.03.080177.000323.

Sabou, J. and Klein, S. (2016) 'How Virtual and Technical Communities Can Contribute to U.N. Led Humanitarian Relief Operations - Boundary Spanning and The Exploration of Collaborative Information Practices', in PACIS 2016 Proceedings, p. 17.

Sabou, J. and Videlov, S. (2016) 'An analysis on the role of trust in digital humanitarian 
actor networks', in Information Systems for Crisis Response and.

Standby Task Force (2011) Why We Need a Disaster 2.1 Report. Available at: http://www.standbytaskforce.org/2011/04/06/why-we-need-a-disaster-2-1-report/ (Accessed: 19 April 2017).

Starbird, K. (2013) 'Delivering Patients to Sacré Coeur : Collective Intelligence in Digital Volunteer Communities', Proceedings of the Conference on Human Factors in Computing Systems (CHI), pp. 801-810. doi: 10.1145/2470654.2470769.

Starbird, K., Muzny, G. and Palen, L. (2012) 'Learning from the Crowd: Collaborative Filtering Techniques for Identifying On-the-Ground Twitterers during Mass Disruptions', Proceedings of 9th International Conference on Information Systems for Crisis Response and Management, ISCRAM, 2011(April), pp. 1-10.

Starbird, K. and Palen, L. (2013) 'Working and sustaining the virtual "Disaster Desk", in Proceedings of the 2013 conference on Computer supported cooperative work - CSCW ' 13. New York, New York, USA: ACM Press, pp. 491-502. doi: 10.1145/2441776.2441832.

Tapia, A. H., Moore, K. a and Johnson, N. J. N. (2013) 'Beyond the Trustworthy Tweet: A Deeper Understanding of Microblogged Data Use by Disaster Response and Humanitarian Relief Organizations', in Proceedings of the 10th International ISCRAM Conference, pp. 770-779. doi: 10.1145/1978942.1979102.

Weinandy, T. (2016) Volunteer and Technical Communities in Humanitarian Response: Lessons in Digital Humanitarianism from Typhoon Haiyan, UN Chronicle. Available at: http://www.oecd-ilibrary.org/content/article/f22a27d6-en (Accessed: 15 March 2017). 


\section{Appendices}

\section{Appendix 1}

Table 1. Summary of the disasters used in the study

\begin{tabular}{|c|c|c|c|c|}
\hline \multirow{2}{*}{$\begin{array}{l}\text { EVENTS } \\
\text { (TYPE) }\end{array}$} & \multirow{2}{*}{$\begin{array}{l}\text { ANALYSIS } \\
\text { PHASE }\end{array}$} & \multicolumn{2}{|l|}{ SOCIAL UNITS } & \multirow[t]{2}{*}{ RESPONSE } \\
\hline & & COUNTRY & CONTINENT & \\
\hline Dam Spillage & Phase 1 & USA (Oroville) & North America & Red \\
\hline \multirow{3}{*}{ Earthquake } & Phase 1 & Japan (Kumamoto) & Asia & Red \\
\hline & Phase 1 & Ecuador & South America & Red \\
\hline & Phase 1 & Italy & Europe & Yellow \\
\hline \multirow{4}{*}{ Explosion } & Phase 1 & Belgium (Brussel) & Europe & Green \\
\hline & Phase 1 & Turkey (Istanbul) & Asia & Green \\
\hline & Phase 1 & Manchester (UK) & Europe & Green \\
\hline & Phase 2 & Westminster (UK) & Europe & Green \\
\hline \multirow{3}{*}{ Flood/Landslide } & Phase 1 & Sri Lanka & Asia & Green \\
\hline & Phase 1 & Peru & South America & Yellow \\
\hline & Phase 1 & USA (Louisiana) & North America & Red \\
\hline Special project & Phase 1 & Burundi & Africa & Purple \\
\hline \multirow{3}{*}{ Severe weather } & Phase 1 & Fiji Tropical Cyclone & Oceania & Green \\
\hline & Phase 1 & USA (Oklahoma) & North America & Green \\
\hline & Phase 2 & Hurricane Irma (USA) & North America & Red \\
\hline \multirow{2}{*}{ Wild fire } & Phase 1 & Canada (Fort McMurray) & North America & Yellow \\
\hline & Phase 1 & Chile & South America & Green \\
\hline Legend & \multicolumn{2}{|c|}{ Combined participation with the observation } & \multicolumn{2}{|c|}{ Observation only } \\
\hline
\end{tabular}




\section{Appendix 2}

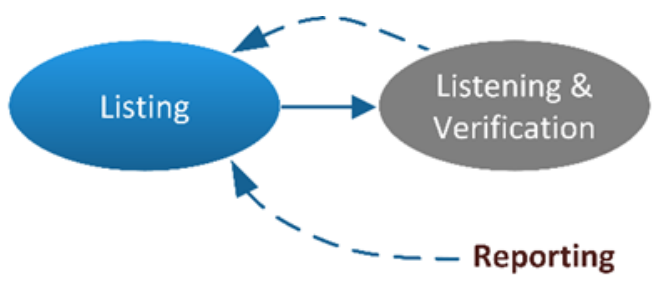

Figure 3. Illustration of the 'Listing' phase.

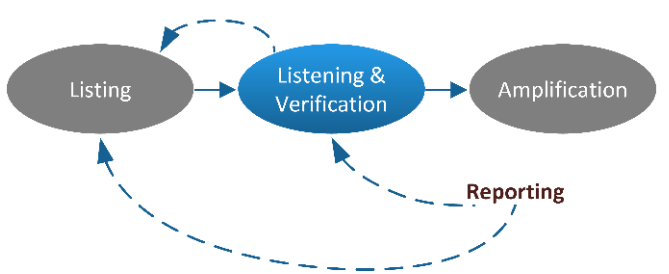

Figure 4. Illustration of the 'Listening \& Verification' phase.

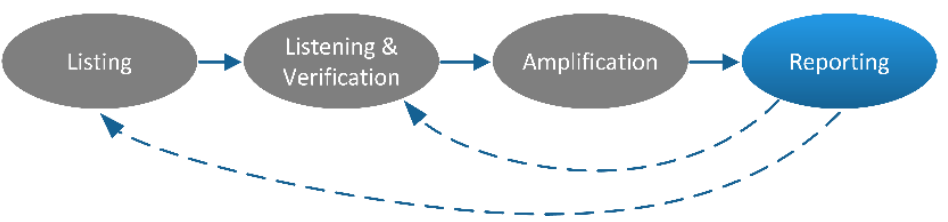

Figure 5. Illustration of the 'Reporting' phase.

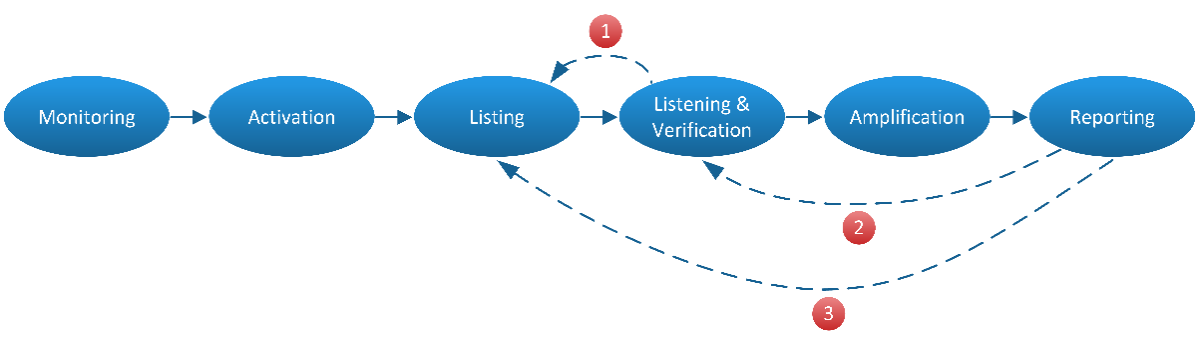

Figure 6. Process workflow. 